\title{
High-Performance Micro Actuators with Applications
}

\author{
Ridha Ben-Mrad \\ Department of Mechanical \& Industrial Engineering, University of Toronto \\ 5 King's College Rd., Toronto, Ontario, M5S 3G8 \\ rbenmrad@mie.utoronto.ca
}

\begin{abstract}
MEMS actuators with high performance are needed for a large number of emerging applications including manipulation of lenses and imaging sensors for adaptive optics and focusing/zooming and optical image stabilization in miniature cameras, micromanipulators, sensing for autonomous vehicles and vector display for HUD in automotive systems and many others. These micro actuators offer varying requirements in terms of stroke, speed, accuracy, reliability, linear versus rotational motion and a number of other requirements. The talk will be presenting novel MEMS actuator platforms with a focus on micro-electrostatic technology that offer large stroke, generate large forces per unit area and a number of degrees of freedom. Different implementations of these micro-actuators are shown and their use for developing a number of applications including 3D micro-mirrors for vector display and automotive head up display, and autofocus and optical image stabilization in miniature cameras.
\end{abstract}

